
allemande

51-1 | 2019

La République démocratique allemande dans l'espace public européen (1949-2018)

\title{
Europäische Institutionenöffentlichkeit - Der Weg zur Mitgliedschaft der DDR bei Icomos (International Council on Monuments and Sites) 1964
} bis 1969

Bianka Trötschel-Daniels

\section{OpenEdition Journals}

Édition électronique

URL : https://journals.openedition.org/allemagne/1552

DOI : 10.4000/allemagne. 1552

ISSN : 2605-7913

Éditeur

Société d'études allemandes

Édition imprimée

Date de publication : 2 juillet 2019

Pagination : $99-112$

ISSN : 0035-0974

Référence électronique

Bianka Trötschel-Daniels, „Europäische Institutionenöffentlichkeit - Der Weg zur Mitgliedschaft der DDR bei Icomos (International Council on Monuments and Sites) 1964 bis 1969", Revue d'Allemagne et des pays de langue allemande [Online], 51-1 | 2019, Online erschienen am: 02 Juli 2020, abgerufen am 02 Juni 2022. URL: http://journals.openedition.org/allemagne/1552 ; DOI: https://doi.org/10.4000/ allemagne. 1552 


\title{
Europäische Institutionenöffentlichkeit - Der Weg zur Mitgliedschaft der DDR bei Icomos (International Council on Monuments and Sites) 1964 bis 1969
}

\author{
- Bianka Trötschel-Daniels *
}

\section{Forschungsfrage und Quellenlage}

Dieser Beitrag zeichnet den Weg nach, den die verantwortlichen Denkmalpfleger in der DDR seit den frühen 1960er Jahren gingen, um sich auf europäischer Ebene zu positionieren. Es werden die Bemühungen um eine Mitgliedschaft der DDR bei Icomos, dem Internationalen Rat für Denkmale und Plätze (International Council on Monuments and Sites) aufgezeigt, deren Erfolg letzten Endes von der Kumulation sich verändernder politischer Einstellungen abhing. Die wichtigen und entscheidenden Vorarbeiten zur Aufnahme fanden allerdings bereits seit den frühen 1960er Jahren statt.

Im Folgenden wird der Begriff „Europäische Öffentlichkeit“ weniger in Bezug auf eine Medien- oder zivilgesellschaftliche Öffentlichkeit, sondern vor allem im Hinblick auf eine Institutionenöffentlichkeit verwendet. Es geht um die Teilhabe von Vertretern eines Landes auf europäischer Ebene in einem institutionalisierten Rahmen, den beispielsweise nichtstaatliche Organisationen bieten können. Innerhalb dieser Organisationen bilden sich Räume des Austausches, der Kommunikation und der Begegnung, etwa auf Kongressen und Tagungen, die einerseits für das Fortkommen der Akteure in ihren nationalstaatlichen Kontexten relevant sind, also zurück wirken auf die nationalstaatliche Ebene. Diese Nationalstaaten können in Europa verortet sein und bilden daher in ihrer Nationalstaatlichkeit einen Teil europäischer Öffentlichkeit. Andererseits bilden sich auf den Tagungen neue Netzwerke und Beziehungen auf einer genuin europäischen Ebene, die sich für die Zeit der Kongresse temporär ergibt. Die Mitgliedschaft bei Icomos bot für die DDR (wie für die anderen Mitglieder auch) die Möglichkeit, sich

* Doktorandin, Kollegiatin im DFG-Graduiertenkolleg „Identität und Erbe“ an der Bauhaus-Universität Weimar, Fakultät Architektur und Urbanistik. 
auf einer fachlichen Ebene mit den Kollegen aus den anderen Staaten auszutauschen und etwa den neusten Stand von Technik und Forschung zu diskutieren. Darüber hinaus ging es um Teilhabe an jener europäischen Öffentlichkeit, die auf den Kongressen und in den Publikationen von Icomos generiert wird. Wie hochgradig politisch diese Teilhabe an der europäischen Öffentlichkeit auf dem Gebiet der Denkmalpflege war, wird anhand des Weges aufgezeigt, den die Vertreter der DDR-Denkmalpflege zurücklegen mussten, von der Vereinbarung im Jahr 1964 eine internationale Denkmalpflegevereinigung zu gründen, bis zur Aufnahme bei Icomos im Dezember 1969.

Dem Beitrag liegen Akten aus den Beständen des Bundesarchivs (BArch) in BerlinLichterfelde, des Politischen Archivs des Auswärtigen Amtes (welches auch die Akten des Ministeriums für Auswärtige Angelegenheiten der DDR (MfAA) beherbergt) sowie des Bundesarchivs in Koblenz zugrunde. Er gibt einen Zwischenstand laufender Forschungen wieder und erhebt keinen Anspruch auf Vollständig- oder Endgültigkeit der bisherigen Ergebnisse. Die Rolle von Icomos als Akteur während des Kalten Krieges ist noch nicht aufgearbeitet. 2017 gab das Deutsche Nationalkomitee eine Quellensammlung mit einigen Dokumenten heraus, die für die Auseinandersetzung Material und Anregung bieten soll. Einen ersten Beitrag zur Aufarbeitung leistete der Beitrag von Aurélie Elisa Gfeller bei einer Tagung $2015^{(1)}$.

\section{Denkmalpflegerisches Bewusstsein als europäische Bewegung in den 1960er Jahren}

Die Sorge um und das Bewusstsein für das baukulturelle Erbe war in den 1960er und 1970er Jahren ein Phänomen europäischen Ausmaßes. Schon 1963 hatte der Österreicher Ludwig Weiß, Mitglied des Kultur- und Wissenschaftskomitees im Europarat, einen Bericht vorgelegt, in dem er die Bedrohung für das kulturelle Erbe in Europa und die Notwendigkeit zu handeln, aufzeigte ${ }^{(2)}$. Vor allem das baukulturelle Erbe wurde durch das neue Leitbild der autogerechten Stadtplanung, durch großflächige Wohnbebauung am Stadtrand bei gleichzeitiger Vernachlässigung der historischen Bausubstanzen in den Innenstädten bedroht. Auch die von einigen als maßstabslos empfundenen Platzgestaltungen, modern interpretierte neue Bauaufgaben, etwa Stadthallen, Bürohochhäuser und Universitätsgebäude, trugen zur „Unwirtlichkeit unserer Städte“ bei, um den Titel eines vom Autor so bezeichneten ,Pamphletes des Psychoanalytikers Alexander Mitscherlich aufzugreifen, der 1965 die Missstände in der Stadtplanung, auch unter gesundheitlichen Aspekten, anprangerte ${ }^{(3)}$.

1 Sigrid Brandt (Hg.), Im Schatten des Kalten Krieges, Dokumente und Materialien zur Geschichte von Icomos Deutschland, Berlin, Hendrik Bäßler Verlag, 2017; Aurélie Elisa Gfeller, „Preserving Cultural Heritage Across the Iron Curtain: The International Council on Monuments and Sites from Venice to Moscow, 1964-1978“, in: Ursula Schädler-SAub, Angela Weyer (Hg.), Geteilt - Vereint! Denkmalpflege in Mitteleuropa zur Zeit des Eisernen Vorhangs und heute, Petersberg, Michael Imhof Verlag, 2015, S. 115-121.

2 Maren Fürniß, „Die Kampagne des Europarates für das Europäische Denkmalschutzjahr 1975 Entstehungsgeschichte, Ziele und Umsetzung“, in: Michael FALSER, Wilfried Lipp, Eine Zukunft für unsere Vergangenheit. Zum 40. Jubiläum des Europäischen Denkmalschutzjahres (1975-2015), Berlin, Hendrik Bäßler Verlag, 2015, S. 73.

3 Alexander Mitscherlich, Die Unwirtlichkeit unserer Städte, Frankfurt am Main, Suhrkamp, 1965. 
Nach dem Bericht des Komiteemitgliedes Weiß empfahl der Europarat die Durchführung eines Maßnahmenplanes für den Schutz des baulichen Erbes in Europa. Er proklamierte das European Architectural Heritage Year, das im deutschen Sprachraum als ,Europäisches Denkmalschutzjahr firmierte. Es fand 1975 seinen Abschluss mit der Deklaration von Amsterdam. Von 1960 bis 1975 bildeten sich überall in Europa Organisationen zur Pflege des kulturellen Erbes. Heute vertritt allein der 1963 in Paris gegründete Dachverband ,Europa Nostra' die Interessen von über 400 Nichtregierungsorganisationen und Privatpersonen gegenüber der Europäischen Union, dem Europarat und der Unesco ${ }^{(4)}$.

Die verantwortlichen Politiker in den Nationalstaaten nahmen die Impulse auf, die einerseits von politischen Institutionen wie dem Europarat und der Unesco ${ }^{(5)}$, andererseits von den zivilgesellschaftlichen Gruppen, die für das kulturelle Erbe eintraten, ausgingen. Überall in den europäischen Nationalstaaten setzten Gesetzgebungsaktivitäten ein. 1960 und 1961 wurden in Schweden und in den Niederlanden die ersten Denkmalschutzgesetze dieser Dekade erlassen ${ }^{(6)}, 1962$ gab sich Polen ein neues Denkmalschutzgesetz ${ }^{(7)}$, in Frankreich wurde ebenfalls 1962 das Loi Malraux erlassen, das den Schutz von Baudenkmalen und die Rechte von Denkmalbesitzern verbesserte ${ }^{(8)}$, Dänemark ersetzte 1966 sein Gesetz von 1918, 1973 erarbeitete die Regierung der ČSSR ein Konzept für ein neues Gesetz zum „Schutz von historischen Werten im Bereiche Kultur“, 1976 wurde in der UdSSR ein neues Denkmalschutzgesetz verabschiedet ${ }^{(9)}$. 1978 wurde in Österreich ein neues Denkmalschutzgesetz erlassen ${ }^{(10)}$. Auch traten

4 Zur Deklaration von Amsterdam: www.dnk.de/_uploads/media/149_1975_Europarat_Deklaration_ Amsterdam.pdf; www.europanostra.org/organisation/, letzter Abruf: 15.01.2019.

5 Empfehlung der Unesco vom 19.11.1968 zur Erhaltung von Kulturgut („Recommendation concerning the Preservation of Cultural Property endangered by Public or Private Works", adopted at the 41. plenary meeting, 19.11.1968); Empfehlung Nr. 612 von 1970 (der damals noch sogenannten) Beratenden Versammlung des Europarates.

6 Für Schweden: www.riksdagen.se/sv/dokument-lagar/dokument/svensk-forfattningssamling/lag1960690om-byggnadsminnen_sfs-1960-690, letzter Abruf: 15.01.2019; für die Niederlande: Eva von ENGelberg-Dockal, „Kategorisierung in der niederländischen Denkmalpflege“, kunsttexte.de, Nr. 2 (2005), S. 1-3.

7 Das Gesetz zum Schutz von Kulturgut vom 15.02.1962 (Ustawa z dnia 15 lutego 1962 r. o ochronie dóbr kultury i o muzeach [Dz. U z 1962 r. Nr. 10, poz. 48]) ersetzte die Verordnung über Denkmalpflege vom 6. März 1928 (rozporządzenia Prezydenta Rzeczypospolitej Polskiej z dnia 6 marca 1928 r. o opiece nad zabytkami [Dz. U.z 1928 r. Nr. 29, poz.265]).

8 Loi n 62-903 du 4 août 1962 complétant la législation sur la protection du patrimoine historique et esthétique de la France et tendant à faciliter la restauration immobilière. «Art. 1 - Des secteurs dits secteurs sauvegardés, lorsque ceux-ci présentent un caractère historique, esthétique ou de nature à justifier la conservation, la restauration et la mise en valeur de tout ou partie d'un ensemble d'immeubles, peuvent être créés et délimités [...].» Online unter: www.legifrance.gouv.fr, letzter Abruf: 15.01.2019, benannt nach dem damaligen Kulturminister André Malraux.

9 Für Dänemark: Harald Langberg, „Das neue dänische Denkmalschutzgesetz“, Deutsche Kunst und Denkmalpflege, Nr. 26 (1968), S. 63-67; für die Tschechoslowakei: Emanuel Hrusk A, „Zur neuen Konzeption des Denkmalschutzes in der SSR“, Deutsche Kunst und Denkmalpflege, Nr. 32 (1974), S. 85-91, hier S. 87; für Russland: Dimitrij DAvydov, Das „fremde“ Erbe. Grenzsicherungsanlagen der 1920er1940er Jahre als Gegenstand des Denkmalschutzes in Russland, Münster, MV-Verlag, 2014, S. 46.

10 BGBl. 167/1978, S. 1089, www.ris.bka.gv.at/Dokumente/BgblPdf/1978_167_0/1978_167_0.pdf, letzter Abruf: 15.01.2019. 
neun von elf Denkmalschutzgesetzen der Länder in der Bundesrepublik Deutschland zwischen 1971 und 1978 in $\mathrm{Kraft}^{(11)}$.

\section{Lage der Denkmalpflege in der DDR in den 1960er Jahren}

Die Denkmalpflege in der DDR war in den 1960er Jahren auf der denkmalpflegerisch-praktischen Ebene geprägt von typischen Aufgaben der Instandhaltungs- und Wiederaufbaumaßnahmen von teilweise noch kriegszerstörten Kirchen, Burgen, Schlössern, Herrenhäusern und Profanbauten ${ }^{(12)}$. Im Laufe der 1960er Jahre kam es allerdings auch zu machtpolitischen Auseinandersetzungen, die an Denkmalen, insbesondere an Kirchenbauten, ausgetragen wurden. Die ikonischen Sprengungen der intakten Universitätskirche in Leipzig und der Ruine der Garnisonkirche in Potsdam im Jahr 1968 prägen das Bild vom Umgang „der DDR“ mit ihrem Denkmalbestand ${ }^{(13)}$. Die Kärrnerarbeit der Denkmalpfleger in der DDR, die mit eingeschränkten Mitteln und trotz manchen Unverständnisses auf politischer Ebene, für den Erhalt der Baudenkmale eintraten, droht von machtpolitischen Gesten einerseits und vom schlechten Zustand der Altbauquartiere zum Zeitpunkt der Wende andererseits überlagert zu werden.

Bei Literatur zur Denkmalpflege der DDR können drei Zugänge unterschieden werden: Zum einen Zeitzeugenberichte, bei denen Denkmalpfleger aus der DDR aus heutiger Sicht über ihre damalige Arbeit, (Miss-)Erfolge und Rahmenbedingungen der Denkmalpflege berichten ${ }^{(14)}$. Zum anderen recherchiert eine neue Generation von Wissenschaftlern heute zur Denkmalpflege in der DDR ${ }^{(15)}$. Ihre Quellen sind auch die

111958 schon hatte Schleswig-Holstein sein Denkmalschutzgesetz verabschiedet. Nordrhein-Westfalen verabschiedete sein Denkmalschutzgesetz zwar erst 1980, gearbeitet wurde daran allerdings ebenfalls seit 1976, vgl. LT-Drs. NW 8/635, 09.02.1976.

12 Für Sachsen umfangreich dokumentiert in: Heinrich Magirius, LANDESAmt für DenKMALPFLege SACHSEn (Hg.), Die Geschichte der Denkmalpflege Sachsens 1945-1989, Dresden, Sandstein Verlag, 2010, insbesondere S.65-145 für die 1960er und 1970er Jahre, S. 141 Fn. 6 und Fn. 7 mit Hinweisen auf die Inventare, die in den einzelnen Arbeitsstellen zu DDR-Zeiten erstellt wurden; Jörg HasPEL, Hubert Staroste, Landesdenkmalamt Berlin (Hg.), Denkmalpflege in der DDR, Rückblicke, Berlin, Nicolai Verlag, 2014.

13 Zum Umgang mit kriegszerstörten Kirchenbauten in der DDR: Henriette von Preuschen, Der Griff nach den Kirchen, Ideologischer und denkmalpflegerischer Umgang mit kriegszerstörten Kirchenbauten in der DDR, Worms, Wernersche Verlagsgesellschaft, 2011, insbesondere für die 1960er Jahre S. 193199. Zu anderen Umgangsformen mit Kirchen: Eva Schäfer, Umnutzung von Kirchen. Diskussionen und Ergebnisse seit den 1960er Jahren, ein Vergleich zwischen den Niederlanden und DDR, Weimar, Bauhaus-Universitätsverlag, 2018, insbesondere Fallbeispiele aus der DDR S.214-234.

14 Haspel/Staroste/Landesdenkmalamt Berlin (Hg.), Rückblicke (Anm. 12); Magirius/Landesamt für Denkmalpflege Sachsen (Hg.), Sachsen (Anm. 12).

15 Forschungen zur Denkmalpflege existieren bereits für Sachsen, Sachsen-Anhalt und Thüringen: Sigrid BRANDT, Geschichte der Denkmalpflege in der SBZ/DDR. Dargestellt an Beispielen aus dem sächsischen Raum 1945-1961, Berlin, Lukas Verlag, 2003; Sandra KeLtsCH, Stadterneuerung und städtebauliche Denkmalpflege in der DDR zwischen 1970 und 1990. Dargestellt an der Entwicklung von Denkmalstädten in Sachsen-Anhalt, Diss., Leipzig, 2012; Katja WüLlner, Hinter der Fassade. Das institutionelle System der Denkmalpflege in der DDR untersucht am Beispiel der thüringischen Städte Erfurt, Weimar und Eisenach, Diss., Cottbus, 2015 sowie die Dokumentation der Tagung Ursula Schädler-Saub, Angela Weyer (Hg.), Geteilt - Vereint! (Anm. 1). Aktuell noch nicht abgeschlossene 
zeitgenössischen Berichte der Denkmalpfleger in den Zeitschriften der DDR, die für einen Überblick über Vorhaben und Projekte der Denkmalpflege in den 1960er und 1970 er Jahren in der DDR konsultiert werden können ${ }^{(16)}$.

Die Jahre von 1961 bis 1975 waren für die Denkmalpflege in der DDR auf verwaltungsorganisatorischer und rechtlicher Ebene eine umbruchreiche Zeit: 1961 löste, kurz nach dem Bau der Mauer, die Verordnung über die Pflege und den Schutz der Denkmale die bisherige, im europäischen Vergleich sehr frühe Verordnung von 1952 $\mathrm{ab}^{(17)}$. Als Denkmal galten ab 1961

„solche Werke der Baukunst und des Städtebaus, der bildenden Kunst und des Kunsthandwerks, der Gartenkunst und der Technik, deren Erhaltung wegen ihrer künstlerischen, geschichtlichen oder wissenschaftlichen Bedeutung im Interesse von Staat und Gesellschaft liegt“" (vgl. \$2 Abs. 1 Denkmal-VO 1961).

Der Denkmalbegriff unterschied sich in den Bedeutungskategorien künstlerisch, geschichtlich und wissenschaftlich nicht von seinem Vorgänger aus der Verordnung 1952, die stark an das Sächsische Heimatschutzgesetz von 1934 angelehnt war, und auch nicht von den Regelungen, die im Laufe der nächsten Jahre in der Bundesrepublik verabschiedet werden sollten ${ }^{(18)}$. Er griff aber, und das war eine Besonderheit der DDR-Denkmalpflege, die Gartendenkmale und die technischen Denkmale ausdrücklich auf. Die Verordnung reagierte endlich auf die verwaltungsorganisatorischen Neuerungen aus dem Jahr 1952, in denen die Länderstruktur zugunsten von Bezirken aufgelöst worden war ${ }^{(19)}$. Die Verordnung passte die Rechtsgrundlagen der Denkmalpflege der Wirklichkeit an, indem sie nicht mehr auf die Landesämter für Denkmalpflege rekurrierte, sondern die Räte der Kreise und Bezirke als Verantwortliche für Schutz und Pflege der Denkmale nannte. Von dem vorgesehenen Listensystem aus der Verordnung von 1952, wonach nur als Denkmal galt, was in konstitutive Listen eingetragen war, verabschiedete man sich in der Verordnung von 1961; ein Denkmal war, was die gesetzlichen Voraussetzungen erfüllte. Als fachwissenschaftliche Einrichtung wurde das Institut für Denkmalpflege nun in der Verordnung verankert. Es agierte über seine fünf Arbeitsstellen in Dresden, Halle, Schwerin, Berlin und Erfurt (wiedererrichtet 1963). Leiter der Denkmalpflege in der DDR war der Generalkonservator. Von 1961 bis 1986 hatte der Architekt Ludwig Deiters diese Stellung inne. Als

Projekte bearbeiten Franziska Klemstein, Denkmalpflege zwischen System und Gesellschaft. Netzwerke der Denkmalpflege im Sozialismus (Arbeitstitel) an der TU Berlin; Luise HeLAs, Ehrenamtliche zivilgesellschaftliche Akteure in der Denkmalpflege der DDR und ihre Bedeutung für die Identitäts- und Erbebildung in Dresden 1965-1990 (Arbeitstitel) und Bianka Trötschel-Daniels, Das Denkmalpflegegesetz der DDR von 1975 (Arbeitstitel), beide an der Bauhaus-Universität Weimar.

16 Zeitschriften: Deutsche Architektur (1952-1990, ab 1974 umbenannt in: Architektur der DDR), Denkmalpflege in der Deutschen Demokratischen Republik (1975-1980).

17 1952: Verordnung zur Erhaltung und Pflege der nationalen Kulturdenkmale, GBl. Nr. 84, S. 514-515; 1961: Verordnung über die Pflege und den Schutz der Denkmale, GBl. II, Nr. 74, S. 475-477.

18 Bianka Trötschel-Daniels, „Kann denn Beton Denkmal sein? Zum Denkmalbegriff im Denkmalpflegegesetz der DDR von 1975“, in: Tino Mager, Bianka Trötschel-Daniels (Hg.), BetonSalon. Neue Positionen zur Architektur der späten Moderne, Berlin, Neofelis Verlag, 2017, S. 127-138, $129 \mathrm{ff}$.

19 Gesetz über die weitere Demokratisierung des Aufbaus und der Arbeitsweise der staatlichen Organe in den Ländern der Deutschen Demokratischen Republik, GBl. 1952, Nr. 99, S. 613-614. 
Leiter des Instituts für Denkmalpflege nahm er sowohl fachliche als auch politische Aufgaben wahr. Er verband die Arbeitsstellen und das Ministerium für Kultur (und nicht etwa das Ministerium für Bauwesen), dem die Denkmalpflege zugeordnet war.

Obwohl die DDR kein Mitglied des Europarates war ${ }^{(20)}$, wurden internationale Aktivitäten - nicht nur auf dem Gebiet der Pflege des kulturellen Erbes - wahrgenommen. Insbesondere über die Mitgliedschaft in Nichtregierungs- und Sonderorganisationen bemühte sich die DDR um eine Teilhabe am internationalen Geschehen ${ }^{(21)}$.

Eine der einflussreichsten Nichtregierungsorganisationen für die Denkmalpflege ist Icomos. Ludwig Deiters hatte schon früh ein Interesse, seine Kollegen aus der DDR bei Icomos zu vernetzen. Seine Anstrengungen waren primär fachlicher, nicht politischer Natur. Die durch eine Mitgliedschaft bei Icomos zu erreichende internationale Aufmerksamkeit zu einer Zeit, in der die völkerrechtliche Anerkennung der DDR noch weit entfernt war (sie wurde erst 1973 bei der UNO aufgenommen), half ihm dennoch bei der Argumentation gegenüber dem Ministerium für Kultur. Entsprechend stolz berichtete er 2014 von dem Erfolg, den die Aufnahme der DDR bei Icomos damals bedeutete:

„Bald darauf erreichten wir die Mitgliedschaft des Icomos-Nationalkomitees der DDR im Internationalen Rat, noch vor der völkerrechtlichen Anerkennung der DDR. Das hat uns nachhaltig die staatliche Unterstützung unserer internationalen Arbeit gesichert “(22).

\section{Die Mitgliedschaft der DDR bei Icomos: ein langer Weg}

Auch in der zeitgenössischen Presse wurde die Nachricht, dass das Icomos-Nationalkomitee der DDR von der Vollversammlung in Paris am 5. Dezember 1969 einstimmig als Mitglied aufgenommen wurde, verbreitet. Das Leitmedium Neues Deutschland berichtete am 10. Dezember 1969 unter der Überschrift „Komitee für Denkmalpflege der DDR in Unesco-Organisation“ von der Aufnahme beim Internationalen Rat für Denkmalpflege ${ }^{(23)}$. Diese Meldung verdeutlicht die Tragweite dieses Schrittes: Die DDR war mit dem Beitritt zu Icomos bei einer Organisation Mitglied geworden, die einen Exekutivstatus bei der Kulturorganisation der UN, der Unesco, innehatte. Die Aufnahme bei Icomos trüge dazu bei, dass die „bedeutenden denkmalpflegerischen Leistungen in der DDR auf diesem Wege internationale Anerkennung“ fänden, so der Tenor der Meldung. Diesem Bericht war ein fast fünf Jahr andauernder Prozess vorausgegangen.

Im Mai 1964 wurde auf dem zweiten Kongress der Architekten und Denkmalpfleger in Venedig in einer von 13 Resolutionen vereinbart, dass ein Internationaler Rat für

20 Neben den neun Ländern der EWG (sechs Gründungsstaaten BeNeLux, Frankreich, Italien und Deutschland, sowie offiziell ab dem 01.01.1973 Irland, Dänemark und dem Vereinigten Königreich) waren auch Norwegen, Schweden, Türkei, Griechenland, Island, Österreich, Zypern, die Schweiz und Malta Mitglied des Europarates.

21 Wilhelm Bruns, „Politik der selektiven Mitgliedschaft. Das Verhältnis der DDR zu den UN-Sonderorganisationen, insbesondere der Unesco“, Vereinte Nationen, Nr. 5 (1978), S. 154-159.

22 Ludwig Deiters, „Das Institut für Denkmalpflege in der DDR - Erinnerungen und Reflexionen“, in: Haspel/Staroste/Landesdenkmalamt Berlin (Hg.), Rückblicke (Anm. 12), S. 16-46, hier S. 23.

23 ADN, „Komitee für Denkmalpflege der DDR in Unesco-Organisation“, Neues Deutschland, 10.12.1969, S. 4 . 
Denkmalpflege gegründet werden solle. Ein Jahr später, im Juni 1965, kam es schließlich in Polen zur offiziellen Gründung dieses Rates.

Ludwig Deiters, politisch und fachlich verantwortlich für die Denkmalpflege in der DDR, bemühte sich seit Beginn des Jahres 1964 um eine Mitgliedschaft der DDR bei der damals noch zu gründenden Vereinigung. Deiters hatte von rumänischen und tschechoslowakischen Denkmalpflegekollegen Anfang 1964 erfahren, dass darüber nachgedacht wurde, eine internationale Denkmalpflegevereinigung zu gründen. Er wandte sich an das Ministerium für Kultur und den dortigen Abteilungsleiter der Abteilung Kulturelle Verbindungen, Roland Feix, mit der Frage, ob die DDR-Denkmalpfleger sich an einer solchen Organisation beteiligen könnten. Neben fachwissenschaftlichen Argumenten, beispielsweise dem Austausch mit den internationalen Kollegen zu Fragen der Erfassung und Restaurierungsmethoden, führte Deiters die politische Dimension einer potentiellen Mitgliedschaft an:

„Gleichzeitig ist der politische Aspekt einer eventuellen Beteiligung der Denkmalpflege der DDR an der Arbeit der Vereinigung [Icomos] nicht zu unterschätzen. Infolge der großen Dichte an kulturhistorisch wertvollen Denkmalen, darunter zahlreichen Bauten und Kunstwerke von Weltgeltung und auf Grund der Bemühungen der Regierung der Deutschen Demokratischen Republik und der verantwortlichen Facheinrichtungen wird die Denkmalpflege in der DDR im Ausland anerkannt“(24).

Welches politische Ausmaß selbst ein Architektenkongress zu Beginn der 1960er Jahre hatte, kann daran abgelesen werden, dass die zum Kongress eingeladenen Vertreter der DDR-Denkmalpflege die Reise zum Kongress nicht antreten konnten: Noch bis zum 26. März $1970^{(25)}$ brauchten alle DDR-Bürger für Reisen in einen NATO-Staat ein sog. Temporary Travel Document (TTD), einen Travel-Pass, der ihnen vom Allied Travel Office (ATO) ausgestellt wurde ${ }^{(26)}$. Diese Behörde wurde von den USA, Frankreich und Großbritannien in Berlin-Schöneberg betrieben. Die Ausstellung dieses temporären Reisepasses wurde den eingeladenen Vertretern der DDR-Denkmalpflege vom ATO verwehrt ${ }^{(27)}$.

An dem Kongress der Architekten und Denkmalpfleger in Venedig 1964 nahmen circa 700 Fachleute teil. Dabei gab es ein „ungefähres Gleichgewicht“ an westund osteuropäischen Delegierten: Hauptsächlich waren vor allem Italien (mit über

24 BArch DR1/15881, Deiters an Feix, 21.02.1964.

25 Hans-Peter Schwarz, Institut für Zeitgeschichte (Hg.), Akten zur Auswärtigen Politik der Bundesrepublik Deutschland (AADP), 1970, Berlin/München, Oldenbourg, 2001, Dok. 1 und 129.

26 Das TTD-System wurde im März 1970 suspendiert. Die drei Westmächte wollten damit zur „Verbesserung der Atmosphäre bei den am 26.3. beginnenden Vier-Mächte-Gesprächen über Berlin beitragen“, vgl. Dok. 129. Schon im Juni 1969 wurde die strenge Handhabung zunächst gelockert und beispielsweise Teilnehmer an Sportveranstaltungen brauchten keinen TTD mehr. Dänemark hatte im Juni 1969 angeregt, die Regelung des TTD vollständig zu suspendieren und setzte sich auch im weiteren Verlauf des Jahre 1969 dafür ein, die TTD-Regelung aufzuheben, vgl. Dok. 1. Ca. 30 Personen suchten tagtäglich das ATO auf, vgl. Der Spiegel, Nr. 35 (1969), S. 49.

27 BArch DR 1/15881, Brief von Feix an MfAA, Frau Kiermeier: „Deiters [...] konnte aber an den bisherigen Beratungen wegen der bekannten Einreiseschwierigkeiten nicht teilnehmen“, 16.02.1965. Sigrid BRANDT, „Internationale Grundsatzpapiere der städtebaulichen Denkmalpflege, Eine Analyse im Vergleich zu städtebaulichen Entwicklungen“, Österreichische Zeitschrift für Kunst und Denkmalpflege (ÖZKD), Heft 1/2 (2015): 50 Jahre Charta von Venedig. Geschichte, Rezeption, Perspektiven, S. 51-59, hier S. 51. 
150 Delegierten), Frankreich (90 Delegierten), Österreich, Belgien, Jugoslawien, die Schweiz und die Sowjetunion (mit je über 20 Delegierten) vertreten ${ }^{(28)}$. Die Bundesrepublik, Niederlande, Großbritannien und Spanien entsandten jeweils circa 10 Teilnehmer. Wegen der Schwierigkeiten mit den TTD, fand der Kongress in Venedig ohne die DDR-Vertreter statt.

In Venedig wurde die Entscheidung getroffen, das noch zu gründende Nationalkomitee Polens mit der Ausrichtung der Gründungskonferenz der neuen internationalen Denkmalpflegevereinigung zu betrauen ${ }^{(29)}$. Piero Gazzola, ein italienischer Architekt und federführend für die berühmt gewordene Charta von Venedig verantwortlich, übernahm den Vorsitz des Organisationskomitees für die Gründung der neuen Vereinigung, die erst bei der Gründung 1965 den Namen Icomos erhielt. Dieses Komitee setzte sich aus Denkmalpflegern aus der ganzen Welt zusammen. Der Vorstand bestand aus Vertretern aus UK, Italien, Belgien, Spanien, Polen, und der Schweiz; außerdem waren Syrien, Ägypten, die Bundesrepublik, die USA, Österreich, Nigeria, Indien, Dänemark, Peru, Mexiko, Japan, Griechenland und Tunesien sowie die Tschechoslowakei, USSR, Jugoslawien und Rumänien am Organisationskomitee beteiligt ${ }^{(30)}$.

Deiters war allerdings gut vernetzt und erfuhr kurz nach der Konferenz in Venedig, im Juni 1964 bei einer Tagung in Saarbrücken von seinem Schweizer Kollegen Schmid, dass vorgesehen war, neben offiziellen Ländervertretungen auch einzelne Institutionen und Einzelpersonen als Mitglieder bei Icomos aufzunehmen. Er schöpfte Hoffnung, dass DDR-Vertreter - trotzdem sie nicht in Venedig teilgenommen hatten - bei Icomos über diesen Weg aufgenommen werden könnten. Er berichtete an die Leiterin der Hauptverwaltung Internationale Beziehungen des Ministeriums für Kultur, Irene Gysi, dass Schmid „gerade darin die Möglichkeit [sähe], die Denkmalpfleger der DDR an der internationalen Vereinigung zu beteiligen“(31).

Deiters bemühte sich eingehend, die DDR-Vertreter an der Gründung von Icomos zu beteiligen. Er reiste gemeinsam mit Feix Anfang April 1965 nach Warschau, um „die Anwesenheit von DDR-Vertretern zum Kongress [im Juni zur Gründung des Icomos] sicherzustellen“(32). Er traf bei dieser Reise auf den Leiter der Abteilung Museumswesen, Ptasznik. Dieser machte Deiters auf die Probleme aufmerksam, die sich in Bezug auf eine angestrebte Mitgliedschaft bei Icomos ergaben: Der Vorsitzende des Polnischen Nationalkomitees Stanislaw Lorentz habe in Vorbereitung auf den Gründungskongress von Icomos geäußert, dass „die Beteiligung der DDR diesen Kongreß belasten würde ${ }^{(33)}$. Die Situation war schwierig: Es galt noch die Hallstein-

28 Kerstin Sтамм, „,Il monumento per l'uomo‘, Zur Entstehungsgeschichte der Charta von Venedig im Kontext der europäischen Nachkriegszeit“, ÖZKD (Anm. 27), S. 20-26, hier S. 24.

29 Aleksandra Zaryn, "The first General Assembly of Icomos 1965“, in: Icomos (Hg.), Thirty Years of Icomos, Scientific Journal 1995, S. 1-10, hier S. 3. Die Verbindung über den Eisernen Vorhang hinweg wertet Gfeller als „powerful symbol of pan-European“, A. E. GFELLER, „Preserving Cultural Heritage“ (Anm. 1), S. 116.

30 A. ZARYN, „The first General Assembly of Icomos 1965“ (Anm. 29), S. 1.

31 BArch DR 1/15581, Deiters an Gysi, 13.07.1964.

32 BArch DR 1/15881, Bericht von Feix über die Konsultation in Warschau am 6. und 7. April 1965 bezüglich der Mitarbeit im Icomos, 10.04.1965.

Ebd. 
Doktrin, wonach die Bundesrepublik Deutschland beanspruchte, die einzig legitime Vertretung des deutschen Volkes zu sein ${ }^{(34)}$. Würde die DDR als Mitglied neben dem Nationalkomitee der Bundesrepublik bei Icomos aufgenommen, verstieße dies gegen die praktizierte Hallstein-Doktrin. Deiters vermutete im Mai 1969 wohl nicht ganz falschliegend, dass Werner Bornheim gen. Schilling, der Präsident des bundesdeutschen Nationalkomitees „offensichtlich Anweisung [hat], gegen die Aufnahme der DDR in das Icomos aufzutreten “(35).

Bei einem Treffen zwischen dem 2. Sekretär der Kulturabteilung der Botschaft der DDR in Polen, Günther Tönnies, und Lorentz am 19. Mai 1965 erklärte Lorentz, dass sich das Komitee für die Gründung von Icomos darauf verständigt habe, nur solche Länder an der Gründungsversammlung im Juni teilnehmen zu lassen, die bereits Mitglied bei der Unesco seien ${ }^{(36)}$. Allerdings sei es eine andere Angelegenheit, dass „nach Gründung des Rates den Statuten entsprechend selbstverständlich weitere Mitglieder aufgenommen werden können“. Tönnies und Lorentz einigten sich darauf, dass wenigstens eine Delegation der DDR zur Zeit der Gründungsversammlung in Warschau weilen würde ${ }^{(37)}$. Dieser Delegation gehörte neben Ludwig Deiters auch Hans Nadler, der Leiter der Arbeitsstelle Dresden des Institutes für Denkmalpflege, an. In seinem Bericht an das Ministerium für Kultur rekurrierte Deiters auf die NichtTeilnahme der DDR am Kongress in Venedig 1964 als Grund dafür, dass die DDR kein Gründungsmitglied bei Icomos werden konnte. Er betonte, dass die Mitgliedschaft bei Icomos nicht von einer Mitgliedschaft bei der Unesco abhänge und dass er die Chancen für eine Aufnahme positiv beurteile, da viele der Vertreter bei Icomos die Denkmalpflege der DDR aus eigener Anschauung kennten ${ }^{(38)}$. Doch die Aufnahme des DDR-Nationalkomitees bei Icomos erfolgte erst im Dezember 1969 und damit noch weitere viereinhalb Jahre nach der Gründung.

Bis 1968 ergaben sich keine Veränderungen. Dann kam erneut Bewegung in die Aufnahmeprozedur bei Icomos. Drei wohl (mit-)entscheidende Schritte waren in den Jahren 1968 und 1969 bewältigt worden: Das DDR-Nationalkomitee der Internationalen Museumsvereinigung Icom (International Council of Museums) wurde beim Internationalen Museumsrat aufgenommen, die Hallstein-Doktrin wurde unter der sozialliberalen Regierung Willy Brandts aufgegeben und das MfAA der DDR betonte die Wichtigkeit von Mitgliedschaften der DDR in internationalen Organisationen.

34 Werner KILIAn, Die Hallstein-Doktrin. Der diplomatische Krieg zwischen der BRD und der DDR 19551973, Berlin, Duncker und Humblot, 2001.

35 BArch DR 1/15881, Deiters zum Stand der Bemühungen um die Aufnahme der DDR bei Icomos, 15.05.1969.

36 Aus diesem Grunde nahm auch Portugal nicht am Gründungskongress teil. Portugal wurde erst 1974 Mitglied der Unesco.

37 BArch DR 1/15881, Tönnies an Feix (MfK), 20.05.1965. Nach der Gründungsversammlung in Warschau 1965 berichtete der Präsident des bundesdeutschen Nationalkomitees Bornheim gen. Schilling zu Recht misstrauisch, dass Deiters und Nadler ihm gegenüber erklärten, „zufällig in Warschau anwesend zu sein“. Für ihn habe sich „eine entsprechende Situation“ ergeben, S. BRANDT (Hg.), Im Schatten des Kalten Krieges (Anm. 1), S. 28.

38 BArch DR 1/15881, Deiters Bericht über die Teilnahme an der Tagung des Icomos, 19.07.1965. 


\section{Icom-Mitgliedschaft 1968}

Die genauen Zusammenhänge zwischen der Aufnahme bei Icom und der Aufnahme bei Icomos sind noch nicht gänzlich bekannt. Bisher konnte aus den Akten das Geschehen wie folgt rekonstruiert werden: Icom war 1946 in Paris gegründet worden. 1964 schon war in Leipzig ein Nationaler Museumsrat ins Leben gerufen worden. Er war damals bereits als Vorstufe für die Aufnahme bei Icom gedacht. Dieser Nationale Museumsrat der DDR hatte zu Beginn des Jahres 1968 einen Antrag bei Icom gestellt, als Nationalkomitee anerkannt zu werden ${ }^{(39)}$. Nach Art. 30 der Satzung von Icom war es auch Staaten, die noch nicht bei der Unesco Mitglied waren, möglich, ein Nationalkomitee bei Icom zu assoziieren.

Am 2. April 1968 wandte sich der Präsident des Deutschen Icomos-Nationalkomitees Bornheim gen. Schilling an das Auswärtige Amt. Scheinbar - die bisher eingesehene Überlieferung ist hier lückenhaft - hatte er vom sowjetischen Vizepräsidenten von Icomos, Vladimir Iwanov, gehört, dass der Präsident des Deutschen Icom-Nationalkomitees Hentzen, einem Vorschlag zugestimmt habe, die DDR solle sowohl bei Icom als auch bei Icomos aufgenommen werden. Dieser Brief Bornheims wurde sogleich vom Auswärtigen Amt an das Bundesministerium des Inneren weitergeleitet. Von dort forderte die Oberregierungsrätin Lugge Hentzen auf, den Sachverhalt aufzuklären. Hentzen verteidigte sich und sagte, dass die Behauptung, auf die Bornheim bezugnahm, nicht zutreffe. Er habe keinem Vorschlag über die Annahme der Nationalkomitees, weder in Icom noch in Icomos, zugestimmt. Das Exekutivkomitee von Icom werde aber bei seiner nächsten Sitzung im August 1968 über den Antrag des Nationalen Museumsrates der DDR abstimmen ${ }^{(40)}$.

Im Mai 1968 wandte Deiters sich an Christa Friedrichs in der Abteilung Kulturelle Verbindungen im Ministerium für Kultur. Er war vom Präsidenten des Sowjetischen Nationalkomitees bei Icomos, Halturin, gebeten worden, Materialien zu senden, die für die geplante Aufnahme des DDR-Nationalkomitees bei Icomos nützlich sein konnten. Deiters forderte diese Materialien von Friedrichs an ${ }^{(41)}$. Anstatt diese zu senden, verfasste Friedrichs lediglich eine handschriftliche Notiz, in der sie vermerkte, dass man sich doch „einig“ gewesen sei, „zunächst Aufnahme Icom abzuwarten“(42). Mit der Fachabteilung im Ministerium war dieses Vorgehen scheinbar abgesprochen, nicht jedoch mit dem Generalkonservator, der sich unabhängig von diesem Plan weiterhin für die Aufnahme bei Icomos einsetzte.

Auf der 25. Sitzung des Exekutivkomitees von Icom wurde beschlossen, ein Nationalkomitee der DDR zu gründen. Dies erfolgte am 31. Juli 1968 von Johannes Jahn, dem Direktor des Museums für Bildende Künste in Leipzig, ausgerechnet in München bei der 8 . Icom-Generalkonferenz ${ }^{(43)}$. Bornheim gen. Schilling erfuhr wohl aus der

39 BArch B 106/59688, Schreiben von Hentzen, Präsident des Deutschen Nationalkomitees bei Icom, an das Bundesministerium des Inneren, 29.04.1968. Ebd.

BArch DR 1/15881, Schreiben von Deiters an Friedrichs, 13.05.1968. 
Süddeutschen Zeitung vom 12. August 1968 von der Aufnahme der DDR bei Icom. Sogleich verfasste er einen Brief, dieses Mal direkt an das Ministerium des Inneren (BMI). Er ahnte: „Damit bahnt sich eine Entwicklung an, die auch für Icomos zahlreiche Folgen haben wird.“ Er bat um eine Stellungnahme des BMI, „was die Meinung Ihres Hauses hierzu hinsichtlich der Konsequenzen für Icomos ist“(44). Die Stellungnahme des BMI traf am 18. September 1968 beim Nationalkomitee in Mainz ein: „Bedauerlicherweise“ sei die Nachricht korrekt.

„Welche Konsequenzen sich aus dieser Entscheidung für Icomos ergeben, läßt sich zum gegenwärtigen Zeitpunkt nicht voraussagen. Es ist jedoch im Interesse der Wahrung der gesamtdeutschen Belange zu hoffen, daß sich eine gleichartige Entwicklung hier vermeiden läßt“(45).

\section{September 1969: Alle Zeichen stehen auf Aufnahme}

Ein knappes weiteres Jahr verging, bis Deiters im September 1969 auf einer von Icomos organisierten Tagung in Leningrad das Signal vom Vizepräsidenten Iwanov, sowie vom Präsidenten des Konsultativkomitees Lorentz und dem Präsidenten des Sowjetischen Nationalkomitees Halturin erhielt, dass nun ein guter Zeitpunkt sei, die Aufnahme des DDR-Nationalkomitees zu beantragen. Das Nationalkomitee hatte sich im April 1969 formiert $^{(46)}$. Bei der nächsten Icomos-Vollversammlung, die im Dezember 1969 in Paris stattfinden würde, könnte die DDR aufgenommen werden. Auch die USA hätten zugesagt, „nicht gegen die Aufnahme der DDR“ aufzutreten ${ }^{(47)}$. Nach seiner Rückkehr von der Tagung informierte Deiters sogleich Irene Gysi über die neuen Entwicklungen. Sie wiederum setzte den stellvertretenden Minister Kurt Bork in Kenntnis ${ }^{(4)}$ und erbat die Zustimmung „zu unserer Absicht, in den Icomos einzutreten“. Am selben Tag informierte auch der Minister für Kultur Klaus Gysi den Minister für Auswärtige Angelegenheiten Otto Winzer über die Entwicklungen bei Icomos. In seinem Brief erläuterte Klaus Gysi Arbeit und Organisation von Icomos, betonte, dass auch andere sozialistische Länder bereits Mitglied bei Icomos seien, dass Icomos die Arbeit von Icom ergänze und momentan den Status B bei der Unesco innehabe, aber in nächster Zeit damit zu rechnen sei, dass der Status A genehmigt würde. Darüber hinaus erläuterte Gysi die Dringlichkeit des Antrages:

„Um sehr wahrscheinlichen Aktivitäten von westdeutscher Seite gegen unseren Aufnahmeantrag keinen Spielraum zu lassen und einer negativen Einflußnahme auf das eine oder

www.icom-deutschland.de/client/media/560/18_mitteilungen_end.pdf, letzter Abruf: 15.01.2019, „[...] alle Voraussetzungen zu schaffen, dass für die Museen der DDR die Mitgliedschaft im Icom erwirkt werden kann“, mit Hinweis auf die Hausmitteilung des Ministeriums für Post- und Fernmeldewesen zum Thema Icom vom 09.08.1965, Hausarchiv des Deutschen Historischen Museums, MfDG, rot 1015, unpag.

44 BArch B 106/59688, Schreiben von Bornheim gen. Schilling, Präsident des Deutschen Nationalkomitees bei Icomos, an das Bundesministerium des Inneren, 22.08.1968.

45 Ebd.

46 BArch DR 1/15581, Betrifft: Initiativkomitee Icomos, 26.09.1969.

47 BArch DR 1/15881, Reisebericht von Ludwig Deiters an MfK, Abt. Kulturelle Verbindungen, 22.09.1969.

48 BArch DR 1/15881, Schreiben von Irene Gysi an Stellvertreter des Ministers Kurt Bork, 28.10.1969. 
andere Mitglied des Exekutivkomitees vorzubeugen, erscheint es angebracht, dem Rat der sowjetischen Genossen zu folgen und noch zur Sitzung dieses Gremiums am 5. Dezember 1969 den Antrag auf Aufnahme zu stellen“(49).

Winzer erklärte sich am 10. November 1969 mit dem Vorschlag Gysis einverstanden, betonte aber, dass „nur eine absolut selbständige Mitgliedschaft des Nationalkomitees der DDR mit der vollen staatlichen Bezeichnung akzeptiert" werden könne ${ }^{(50)}$.

Zwischenzeitlich hatte in der Bundesrepublik allerdings der Wechsel zur sozialliberalen Koalition stattgefunden. Mit ihm veränderte sich auch die Politik gegenüber der DDR. In seiner Regierungserklärung vom 20. Oktober 1969 sprach Bundeskanzler Willy Brandt von „zwei Staaten einer Nation“ und gab damit die Hallstein-Doktrin auf. Wie das Beispiel der Aufnahme der DDR beim Internationalen Museumsrat Icom zeigt, konnte auch zu Zeiten, in denen die Hallstein-Doktrin noch angewandt wurde, eine Mitgliedschaft der DDR bei internationalen Organisationen erreicht werden. Inwiefern die Aufgabe der Hallstein-Doktrin sich unmittelbar auf den Fortgang der Aufnahme der DDR bei Icomos auswirkte, bedarf noch weiterer Untersuchungen. Schließlich hatten, wie dargestellt, die Vorbereitungen für die Aufnahme bei Icomos schon lange vor der Aufgabe der Hallstein-Doktrin begonnen.

Zur gleichen Zeit, im November 1969, erarbeitete man im MfAA eine Vorlage für den Ministerrat, in der die Wichtigkeit von Mitgliedschaften der DDR in nichtstaatlichen internationalen Organisationen unterstrichen wurde. Die Mitgliedschaften trügen „,in hohem Maße zur internationalen Anerkennung der DDR bei“.

„Unter Bedingungen, da die DDR noch nicht Mitglied der UNO und ihrer Spezialorganisationen ist, stellt die selbständige Mitgliedschaft von DDR-Institutionen in einer Vielzahl nichtstaatlicher internationaler Organisationen eine anschauliche Zurückweisung der westdeutschen Alleinvertretungsanmaßung dar [... “".

Darüber hinaus gäbe sie die

„Möglichkeit, die Errungenschaften der DDR in Wirtschaft, Wissenschaft, Technik und Kultur in vielfältigen internationalen Gremien, Kongressen usw. darzustellen und andererseits die Leistungen und Forschungsergebnisse anderer Länder für den Aufbau des Sozialismus in der DDR nutzbar zu machen“(51).

Insbesondere seien Mitgliedschaften in solchen Institutionen von Bedeutung, die einen Konsultativstatus bei der UNO und deren Unterorganisationen besäßen. Denn das gebe der DDR gewisse Möglichkeiten, auch mittels nichtstaatlicher internationaler Organisationen ihren Anspruch auf Mitgliedschaft im UNO-System geltend $\mathrm{zu}$ machen und in bestimmtem Maße an Veranstaltungen von UNO-Organen teilzunehmen $^{(52)}$.

49 BArch DR 1/15881, Schreiben von Klaus Gysi an den Minister für Auswärtige Angelegenheiten Otto Winzer, 28.10.1969.

50 BArch DR 1/15881, Schreiben von Otto Winzer an den Minister für Kultur Klaus Gysi, 10.11.1969.

51 MfAA/C/554-75, Blatt 118, Vorlage des MfAA an die Kommission des Ministerrates der DDR für die Gestaltung der Arbeit im Bereich Wissenschaft und Kultur nach Westdeutschland und Westberlin vom 19.11.1969. 
Die Aufnahme bei Icomos erfolgte also zu einer Zeit, in der die politischen Bedingungen sowohl in der Bundesrepublik als auch in der DDR günstig waren. Am 5. Dezember 1969 erfolgte in Paris die Aufnahme des DDR-Nationalkomitees bei Icomos. Die Abstimmung erfolgte einstimmig und damit auch mit den Stimmen der bundesdeutschen Delegation.

\section{Fazit}

Die Denkmalpfleger der DDR agierten in einem europäischen Kontext. Obwohl ihre Arbeit lokal verortet war, entfaltete sie Wirkungen auf internationaler Ebene. Die Anbindung an die europäische Ebene verschaffte den DDR-Denkmalpflegern fachliche Vorteile: sie konnten sich mit den Kollegen austauschen und so am aktuellen Stand der Forschung teilhaben, die Impulse europäischer Einflüsse für die nationale Denkmalpflege fruchtbar machen und die Entwicklungen in der DDR-Denkmalpflege vorantreiben.

Auf politischer Ebene war die Mitgliedschaft bei Icomos ein wichtiges Argument für die DDR-Denkmalpfleger gegenüber den Verantwortlichen im Hinblick auf Ressourcen und Baukapazitäten, da sie Erfolge der DDR-Denkmalpflege auf internationaler Ebene vorstellten und bekannt machten, was wiederum für die Außenwirkung der DDR von Vorteil war.

Immer wieder wurde seitens der Denkmalpfleger mit der außenpolitischen Wirkung der Denkmalpflege argumentiert. Die Denkmalpflege wurde - ähnlich wie der Sport - genutzt, um internationale Aufmerksamkeit zu erzeugen. Der Blick über den nationalstaatlichen Tellerrand, das Agieren in einer europäischen Öffentlichkeit, nutzte den Denkmalpflegern im Land und wurde gleichzeitig von ihnen genutzt, um ihre Interessen durchzusetzen.

Die Denkmalpfleger waren durch ihre Fähigkeiten und Kompetenzen sogar in der Lage, politische Strukturen zu überwinden und so dazu beizutragen, dass die DDR noch vor der offiziellen internationalen Anerkennung Mitglied in wichtigen Fachorganisationen wurde. Der Weg, den das DDR-Nationalkomitee nehmen musste, bis es bei Icomos aufgenommen war, zeigt aber auch, dass die Entscheidung letztendlich nicht allein von den Akteuren in der DDR abhing. Erstens spielte die Beziehung zur Bundesrepublik sowohl auf einer fachlichen Ebene zwischen den Denkmalpflegern, als auch auf politischer Ebene bei Außen- und Innenpolitik eine entscheidende Rolle. Zweitens engagierten sich auch Kollegen aus anderen Ländern für die Aufnahme der DDR. Ihre originäre Nationalstaatlichkeit spielte dabei zwar sicher eine Rolle. So ist es für die DDR jedenfalls von Vorteil gewesen, dass der Vizepräsident von Icomos aus der Sowjetunion stammte. Doch die Akteure agierten im Rahmen einer Institutionenöffentlichkeit auf einer europäischen Ebene. Die bei Icomos entstandene Öffentlichkeit war dabei nicht nur eine, die sich aus der Summe der Nationalitäten der Angehörigen ergab, sondern eine genuin europäische Öffentlichkeit. 


\title{
Résumé
}

Cet article retrace le chemin emprunté en RDA par les conservateurs de monuments depuis le début des années 1960 pour se positionner au niveau européen. Il montre les efforts déployés pour adhérer à l'Icomos (International Council on Monuments and Sites / Conseil international des monuments et des sites), démarche dont le succès dépendit en définitive des multiples changements d'attitude politique à la fin des années 1960. Toutefois, d'importants travaux préparatoires avaient été mis en ouvre dès le début des années 1960 en vue de cette adhésion.

\begin{abstract}
This article traces the path taken by the responsible monument conservators in the GDR since the early 1960s to position themselves at a European level. It shows the efforts for membership at Icomos, the International Council on Monuments and Sites, whose success ultimately depended on the accumulation of changing political attitudes in the late 1960s. However, the important and crucial preparatory work for the recording had already taken place since the early 1960s.
\end{abstract}

